\title{
FIBER OPTIC MAGNETIC FIELD SENSOR USING SPECTRAL MODULATION ENCODING
}

\author{
M. Lequime and C. Meunier \\ BERTIN \& Cie, Optics and Optoelectronics Division \\ BP 22, 13762 Les Milles Cedex, FRANCE \\ H. Giovannini \\ Ecole Nationale Supérieure de Physique de Marseille \\ Domaine Universitaire de St. Jérôme, 13397 Marseille Cedex 13, FRANCE
}

\begin{abstract}
This paper is devoted to the description of a new type of fiber optic magnetic field sensor based on spectral modulation encoding techniques. This device uses a Faraday magneto-optic element as transducer and converts the rotation of the plane of polarization of the light induced by the magnetic field into a small change in the large optical path difference provided by a birefringent coding plate. The use of a broadband source and of an interferometric detector, with an optical path difference matched to the sensor one, allows to reach high resolution and perfect down-lead insensitivity. A dual-wavelength arrangement is proposed to determine the temperature and magnetic fied values with the same probe.
\end{abstract}

\section{INTRODUCTION}

In order to optimize the operation of a railway machine, it can be very useful to monitor the variations of the electric supply current used to drive the motors of such engines in real time. Naturally, the current transducers and, in the same way, the supplying and transmitting lines are submitted to a high level of electromagnetic interferences, which yields critical the use of classical devices (like electrically powered sensor).

The choice of a fiber optic sensor allows to greatly reduce the difficulties rised by such an environment, and provides thus an attractive solution to achieve the entire instrumentation of this kind of vehicle.

For our specific application (current monitoring), the main requirements are the following:

- Full Scale : 0 - 2000 Amps

- Working Range : $0-1000$ Amps

- Accuracy : $5 \%$ of the working range (50 Amps)

- Bandwith : less than $1 \mathrm{kHz}$

- Temperature : $\quad-20{ }^{\circ} \mathrm{C},+70{ }^{\circ} \mathrm{C}$ (storage $-40{ }^{\circ} \mathrm{C},+85^{\circ} \mathrm{C}$ )

This electric current spreads through conducting lines with rectangular $(30 \mathrm{~mm}$ by $10 \mathrm{~mm}$ ) or circular ( $20 \mathrm{~mm}$ diameter) sections.

We have chosen to determine in all cases the electric current values by monitoring the magnetic field which it induces around the conducting line. Taking into account the transverse dimension ( $\mathrm{r}$ ) of the conductor, and using the classical relation between current (I) and magnetic field (B) in such a geometry :

$$
\mathrm{B}=\frac{\mu_{0} I}{2 \pi} \frac{1}{\mathrm{r}}
$$

we can cstimate the magnetic field overall range (about 200 Gauss) and the aimed accuracy (better than 10 Gauss).

The achievment of these performances implies naturally that the selected coding scheme offers a perfect down-lead insensitivity (a change in the fiber optic link does not induce any modification of the parameter value provided by the 
sensor, in its accuracy range) and yields possible the correction of the thermal drifts of the transduction in the useful temperature range (about $100^{\circ} \mathrm{C}$ ) by simple means. Spectral modulation encoding techniques, which connect the measurand to the value of the frequency of a sinusoidal modulation inscribed by the sensor in the spectrum of a broadband source, allow to fulfil all these requirements ${ }^{1-4}$.

\section{GENERAL PRESENTATION OF THE CODING SCHEME}

Spectral modulation encoding techniques are based on the use of three main elements, connected all together through multimode optical fibers : a broadband source, an interferometric transducer and an interferometric demodulator ${ }^{3}$.

The broadband source shall have a smooth wavelength dependent intensity distribution, like the one of a Light Emitting Diode (LED); in first approximation, the spectral intensity distribution of such an incoherent emitter can be described by a gaussian function :

$$
P(\sigma)=\frac{P_{0}}{\sqrt{\pi} \delta \sigma} e^{-\left[\frac{\sigma-\sigma_{0}}{\delta \sigma}\right]^{2}}
$$

where $\sigma_{0}$ is the central wavenumber of the LED, $\delta \sigma$ its spectral bandwith and $\mathrm{P}_{0}$ the total output power launched into the fiber core. An important item for the definition of the whole system is the coherence length of the source $1_{c}$, which can be estimated from the spectral bandwith FWHM $\Delta \lambda$ with the help of the following relation :

$$
l_{c} \approx 1.4 \frac{\lambda_{0}^{2}}{\Delta \lambda}
$$

For a LED emitting around $850 \mathrm{~nm}$, the spectral bandwith FWHM is typically less than $50 \mathrm{~nm}$ and the coherence length of the optical radiation is therefore about $20 \mu \mathrm{m}$.

The sensor is a two beam interferometer, for which the Optical Path Difference (OPD) $\Delta_{S}$ is a function of the measurand $\mathrm{X}$ (in our case, the magnetic field $\mathrm{B}$ ) and greater than the coherence length of the source (at least, two to three times $l_{\mathrm{c}}$ ).

The detector is an interferometric device too, which provides a fixed OPD $\Delta_{\mathrm{d}}$, which is equal to the mean value of $\Delta_{S}(X)$ in the range of variation of the measurand. The total amount of light $\mathrm{S}$ detected at the output of the detector is given by the following relation ${ }^{5}$ :

$$
S=T_{1} T_{2} T_{s} T_{d} P_{0}\left\{1+\frac{1}{2} m_{s} m_{d} e^{-\left[\pi \delta \sigma\left(\Delta_{s}-\Delta_{d}\right)\right]^{2}} \cos 2 \pi \sigma_{0}\left(\Delta_{s}-\Delta_{d}\right)\right\}
$$

where $T_{1}$ and $T_{2}$ are the transmissions of the optical fiber links, $T_{S}$ and $T_{d}$ the transmissions of the interferometers used in the sensor and the detector, $\mathrm{m}_{\mathrm{S}}\left(\right.$ resp. $\mathrm{m}_{\mathrm{d}}$ ) the visibility of the modulation inscribed by the sensor (resp. the detector) in the spectrum of the broadband source.

We have previously developed ${ }^{5}$ an Emission/Detection Unit (the $\mathcal{A C C O R D ^ { \circledR }}$ Module), which is able to detect, with a great accuracy, the OPD mismatch between the sensor and the detector, and thus, after calibration, to provide the value of the measurand X. Naturally, while the phase information is coded into the spectrum of the source, the variations of the transmission of the optical fiber links used to connect together the different elements have no effect on the determination of the measurand (the beat phase value is independent of all $\mathrm{T}$ parameters). 


\section{DESIGN OF THE PROBE}

\section{1 - TRANSDUCTION SCHEME}

The probe shall be based on an interferometric principle and characterized by a simple relation (for example linear or slightly quadratic) between the value of the magnetic field B and the OPD $\Delta_{S}$ created by the transducer. On the other hand, this OPD shall be in accordance with the requirements of the spectral modulation encoding techniques, that is basically two to three times greater than the coherence length of the source.

Three main effects could be theoretically used to achieve the magnetic field transduction : the magnetostrictive effect, the COTTON-MOUTON effect and the FARADAY effect.

The magnetostrictive effect could be used easily by introducing a small magnetostrictive spacer into a FABRYPEROT arrangement : a variation of the magnetic field B would induce a corresponding modification of its optical thickness. Unfortunately, the hysteresis behaviour of this kind of compound prevents from its using for metrologic purposes.

The COTTON-MOUTON effect corresponds to the induction of a linear birefringence in presence of a magnetic field. The amplitude of this birefringence is proportional to the square of the magnetic field (pure quadratic dependence), and consequently the sensitivity at small field is poor.

The last possibility is to use the well-known FARADAY effect, which has the required linear dependence versus the magnetic field. However, the induced birefringence is of circular type, and characterized by a very small delay between the two circular components, that prevents normally its use in a spectral modulation encoding scheme. In order to overcome this difficulty, we have chosen to use a more complex interferometric configuration, in which the field induced circular birefringence is added to a large and stable linear birefringence provided by a coding plate.

\section{2 - DESCRIPTION OF THE PROBE}

The drawing of the probe is presented in Figure 1A, while the Figure 1B specifies the relative orientations of the various birefringent elements ${ }^{6}$.

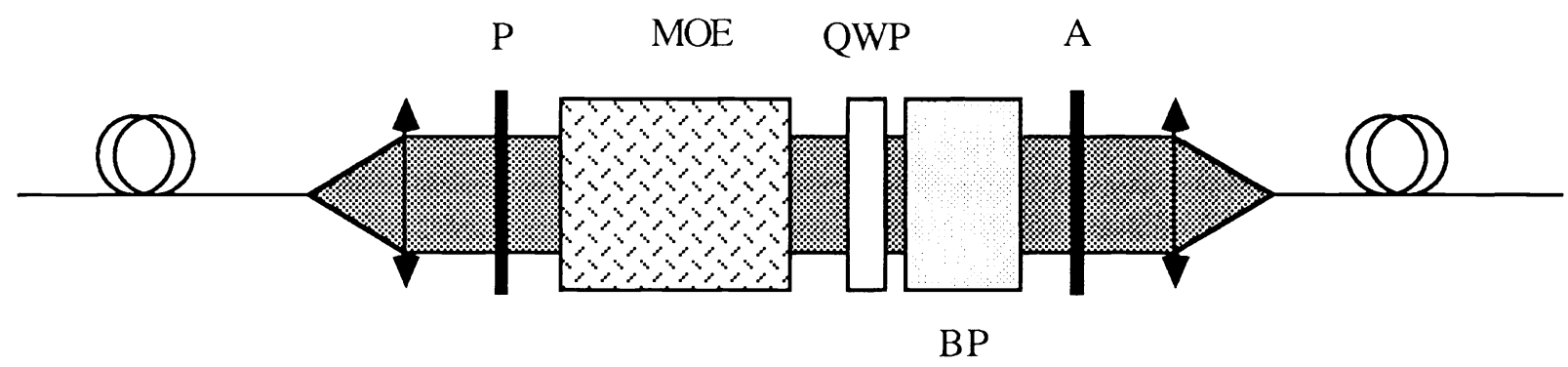

Fig. 1A. Design of the Optical Probe

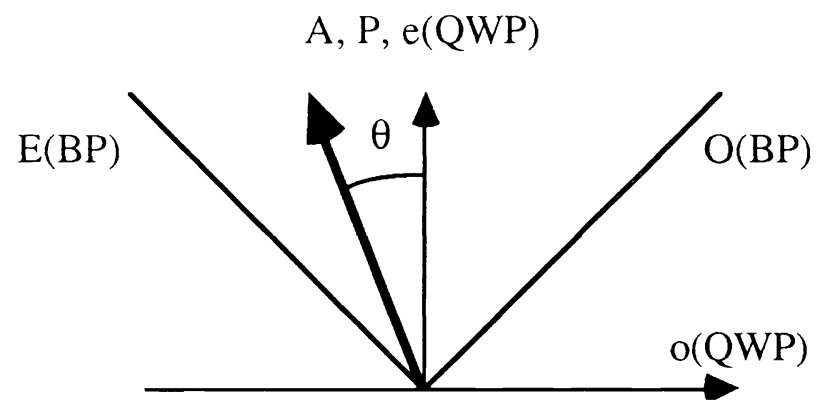

Fig. 1B. Axis relative orientations 
The light provided by the broadband source (a LED centered on $840 \mathrm{~nm}$, with $50 \mathrm{~nm}$ of spectral bandwith FWHM) is launched into a multimode step index fiber, 100 microns core and 140 microns cladding diameters. The extremity of the fiber is precisely positioned at the focal plane of a single lens, in order to obtain a well colimated optical beam. This beam is polarized with the help of a dichroic polarizer P and passes through a SF 57 diamagnetic glass, used as Magneto-Optic Element (MOE). When a magnetic field B is applied along this component, the State Of Polarization (SOP) of the light remains perfectly linear, but is rotated by an angle $\theta$ proportional to $\mathrm{B}$, in accordance with the following relation :

$$
\theta=\mathrm{V} 1 \mathrm{~B}
$$

where 1 is the length of the magneto-optic element and V its VERDET constant. By adding a Quarter Wave Plate (QWP), we transform the circular birefringence induced by the Magneto-Optic Element into a linear birefringence, wich is directly related to the angle between the direction of polarization of the light and the main axis of the Quarter Wave Plate. This variable and small birefringence $( \pm \lambda / 4)$ is added to the static birefringence e $\Delta \mathrm{n}$ provided by a Coding Plate (CP). The probe contains finally an analyser $\mathrm{A}$, with same orientation and constitution as the polarizer. The transmitted beam is focussed by a simple lens and launched into the same type of fiber as previously described (SI 100/140).

\section{3 - SPECTRAL TRANSFER FUNCTION OF THE PROBE}

The transmission spectrum of the probe can be determinated using JONES calculus. Referring to Fig. 1A, the output of the system can be represented by the matrix equation

$$
E^{\prime}(\lambda)=A \cdot B(\lambda) \cdot Q \cdot M \cdot P E(\lambda)
$$

where $E(\lambda)$ and $E^{\prime}(\lambda)$ are the column vectors giving the $x$ and $y$ components of the input and transmitted electric fields, respectively, $P$ and $A$ the matrices of the polarizers, and $M, B(\lambda)$ and $Q$ the matrices respectively associated with the magneto-optic element, the birefringent coding plate and the quarter wave-plate (achromatic retarder). These matrices are given by

$$
\begin{gathered}
P=\left[\begin{array}{ll}
0 & 0 \\
0 & 1
\end{array}\right] \text { and } A=\left[\begin{array}{ll}
0 & 0 \\
0 & 1
\end{array}\right] \\
M=\left[\begin{array}{ll}
\cos \theta & -\sin \theta \\
\sin \theta & \cos \theta
\end{array}\right] \quad \mathrm{B}=\left[\begin{array}{cc}
\cos \Gamma / 2 & -\mathrm{i} \sin \Gamma / 2 \\
-\mathrm{i} \sin \Gamma / 2 & \cos \Gamma / 2
\end{array}\right] \text { and } \mathrm{Q}=\mathrm{e}^{-\mathrm{i} \pi / 4}\left[\begin{array}{ll}
1 & 0 \\
0 & \mathrm{i}
\end{array}\right]
\end{gathered}
$$

where the term $\Gamma(\lambda)=2 \pi \mathrm{e} \Delta \mathrm{n} / \lambda$ describes the optical retardation produced by the birefringent coding plate. Substituting the matrices in the previous equation and using the relation $T(\lambda)=\left|E^{\prime}(\lambda) / E(\lambda)\right|^{2}$, we obtain finally the overall transmittance of the probe :

$$
\mathrm{P}_{\mathrm{c}}(\sigma)=\frac{1}{4} \mathrm{~T}_{\mathrm{c}} \mathrm{P}_{0}(\sigma)\{1+\cos (2 \pi \sigma \mathrm{e} \Delta \mathrm{n}-2 \theta)\}
$$

The sensor OPD $\Delta_{\mathrm{S}}$ is well the sum of both birefringences (circular birefringence of the magneto-optic element and linear birefringence of the coding plate) and a linear function of the magnetic field $\mathrm{B}$, while we obtain finally, by referring to the previous equations :

$$
\Delta_{\mathrm{s}}=\mathrm{e} \Delta \mathrm{n}-\frac{\theta}{\pi} \lambda=\mathrm{e} \Delta \mathrm{n}-\frac{\lambda \mathrm{Vl}}{\pi} \mathrm{B}
$$


The measurement of the phase mismatch between the sensor and the detector will allow to recover the magnetic field value without indetermination, if the induced circular birefringence remains smaller than $2 \pi$ for any field value in the working range of the probe (possible adjustement by the choice of some sensing element features, as thickness or Verdet constant).

\section{EXPERIMENTAL RESULTS}

A functional description of the experimental set-up is presented in Figure 2. The emission unit includes two standard LEDs, with slightly different features :
* LED $1: \lambda_{0}=840 \mathrm{~nm}$
$\Delta \lambda=50 \mathrm{~nm}$
$\mathrm{P}_{0}=560 \mu \mathrm{W}$
* LED $2: \lambda_{0}=890 \mathrm{~nm}$
$\Delta \lambda=60 \mathrm{~nm}$
$\mathrm{P}_{0}=180 \mu \mathrm{W}$

All the experiments described in this paper have been performed with the LED 1, because of its better optical power figure.

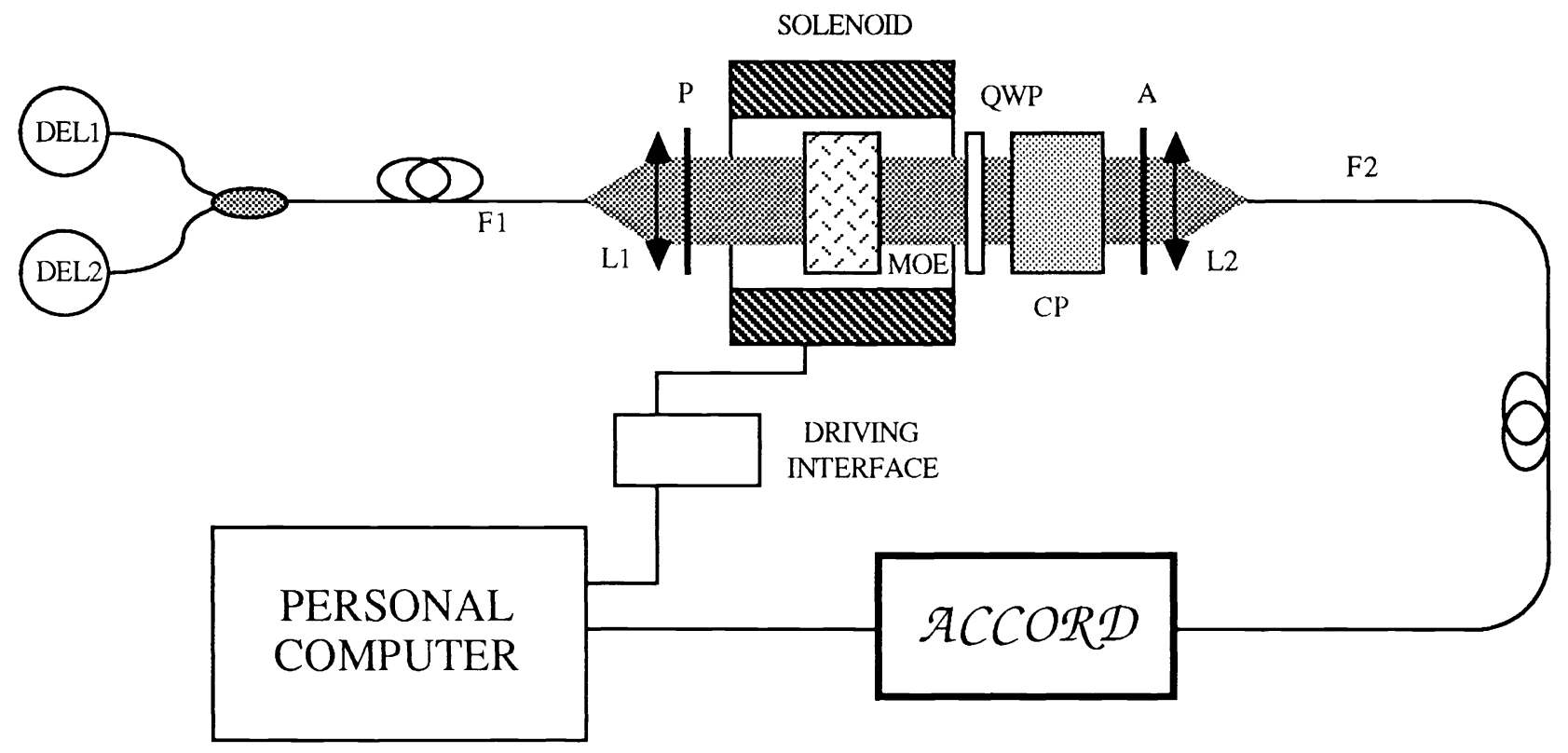

Fig. 2. Experimental set-up

The $\mathrm{L} 1$ and $\mathrm{L} 2$ lenses are standard achromat lenses, $10 \mathrm{~mm}$ focal length. The used fibers are multimode step index fibers, 100 microns core and 140 microns cladding diameters and .22 Numerical Aperture. The Magneto-Optic Element MOE is a $20 \mathrm{~mm}$ diameter and $40 \mathrm{~mm}$ length cylindrical SF57 rod. This glass plate is located at the center of a solenoid, which is supplied by an adjustable DC current (the amplitude of the magnetic field is proportional to the current value, while its direction is defined by the current polarity). An electronic module achieves the interface between the analogic driving signal provided by the Personal Computer $(0-10 \mathrm{~V})$ and the solenoid : the current value can be adjusted in a $0-4 \mathrm{~A}$ range with a great accuracy (12 bits DAC). For the maximum value of the current (4A), the magnetic field at the center of the solenoid reaches 260 Gauss. The Quarter Wave Plate is constituted by a three element achromatic retarder, optimized around $850 \mathrm{~nm}$. In this first experiment, the Coding Plate is a $25 \mathrm{~mm}$ length Quartz cristal ( $\mathrm{e} \Delta \mathrm{n} \approx 220$ microns).

The output fiber $\mathrm{F} 2$ is directly connected to the $\mathcal{A C C O R D ^ { \circledR }}{ }^{\circledR}$ detection module, which contains basically a polarimetric device allowing the extraction of the phase mismatch between the sensor and the receiver with a great accuracy (150 $\mu \mathrm{rads} / \mathrm{NHz}$ ). The values provided by this detection module are processed by a Personal Computer and displayed on a monitor or a plotter. 
Figure 3 shows the experimental results obtained with 250 Gauss magnetic field steps.

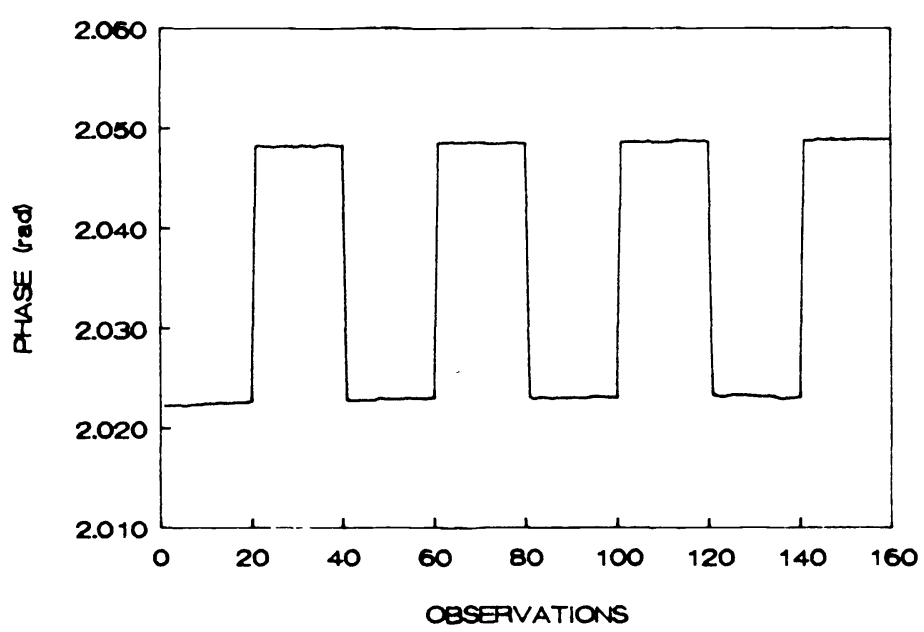

Fig. 3. Experimental records of field induced phase variations

The measured sensitivity is about $100 \mu \mathrm{rads} /$ Gauss, while the expected value is $92 \mu \mathrm{rads} /$ Gauss, using classical SF57 data $^{7}$ at $633 \mathrm{~nm}$ and $1 / \lambda^{2}$ spectral dependence of its Verdet constant. The resolution of this first experimental set-up is thus better than 2 Gauss, at $1 \mathrm{~Hz}$ bandwith.

\section{DISCUSSION}

\section{1 - TEMPERATURE EFFECTS}

According to the expression of the sensor OPD and neglecting the thermal expansion of the birefringent optical plates (MOE and $\mathrm{CP}$ ), the phase sensitivity to a temperature variation of the probe is given by the following relation

$$
\frac{\partial \phi}{\partial \mathrm{T}}=\frac{2 \pi}{\lambda} \mathrm{e} \frac{\partial \Delta \mathrm{n}}{\partial \mathrm{T}}+2 \mathrm{lB} \frac{\partial \mathrm{V}}{\partial \mathrm{T}}
$$

which points out two separate effects :

* a scale factor effect, induced by the thermal dependence of the Verdet constant; for a SF57 glass, the amplitude of this physical property has been measured at $633 \mathrm{~nm}\left(1 / \mathrm{V} \partial \mathrm{V} / \partial \mathrm{T}=1.710^{-4}(\mathrm{~K})^{-1}\right)$. This value allows to fulfil the accuracy requirements for 200 Gauss range and $45 \mathrm{~K}$ thermal excursion (related field error less than 2 Gauss).

* an offset drift effect, induced by the thermal dependence of the static linear birefringence provided by the coding plate. For the experimental configuration (SF57 rod associated with a thick Quartz plate), which combines a relatively poor magnetic field sensitivity for the sensing element and a high thermal sensitivity for the coding element (about $10^{-4}$ ), reaching the aimed accuracy (5\%) supposes that the temperature of the probe remains stable at better than $.01{ }^{\circ} \mathrm{C}$, which can be achieved only during small time periods in laboratory.

In order to eliminate the thermal drifts (scale factor and offset) of such a probe, we should associate two improvements :

- increasing the field sensitivity of the magneto-optic element, with maintaining a small thermal dependence of the 
Verdet constant : this can be achieved by using iron garnets (YIG), or still better, substituted iron garnets (like Ga:YIG), for which the field sensitivity reaches $.1 \mathrm{rads} /(\mathrm{Gauss} . \mathrm{cm})$ and remains stable at better than $5 \%$ in the $20-100{ }^{\circ} \mathrm{C}$ range 8 .

- reducing the thermal sensitivity of the coding element by using an athermal birefringent composite plate ${ }^{9}$, that should allow the reduction of the relative variation factor to less than $10^{-6}(\mathrm{~K})^{-1}$.

\section{2 - DUAL-WAVELENGTH ARRANGEMENT}

On the other hand, the thermal sensitivity of the Coding Plate can be used to determine with the same probe both parameters (temperature and magnetic field) with an improved accuracy.

For this purpose, we can use the two independent informations provided by the dual wavelength arrangement of the Emission/Detection Module (as indicated before, $\mathcal{A C C O R D ^ { \circledR }}$ is basically a two wavelength system). Assuming a standard spectral dependence for the Verdet constant of the sensing element $\left(V=K / \lambda^{2}\right)$, and an achromatic behaviour of the coding plate, we can compute two expressions $\tau$ and $\beta$

$$
\begin{aligned}
& \tau=\lambda_{2}^{2} \phi_{2}-\lambda_{1}^{2} \phi_{1}=2 \pi\left(\lambda_{2}-\lambda_{1}\right) \text { e } \Delta \mathrm{n}(\mathrm{T}) \\
& \beta=\lambda_{2} \phi_{2}-\lambda_{1} \phi_{1}=\frac{2 \mathrm{~K}(\mathrm{~T}) \mathrm{l}}{\Lambda} \mathrm{B} \text { with } \Lambda=\frac{\lambda_{2} \lambda_{1}}{\lambda_{2}-\lambda_{1}}
\end{aligned}
$$

which allow to determine first the value of the temperature $(\tau)$ and secondly the exact value of the magnetic field taking into account the thermal dependence of the scale factor $(\beta)$.

\section{CONCLUSION}

This work demonstrates the possibility to associate FARADAY effect and spectral modulation encoding to achieve the accurate detection of a magnetic field. This new coding scheme allows to design small size extrinsic fiber optic sensor (linear single path arrangement) with a perfect down-lead insensitivity. First experiments performed with SF57 glass rod have demonstrated the efficiency of such an arrangement and confirmed the theoretical sensitivity evaluations. The use of substituted iron garnets as sensing element should allow to reach better thermal properties for the whole probe and to avoid any kind of temperature corrections in a large thermal range (100 degrees).

The experimental validation of the transduction principle has been partially supported by RATP (Régie Autonome des Transports Parisiens).

\section{REFERENCES}

1. Th. Bosselmann and R. Ulrich, "High Accuracy Position-Sensing with Fiber-Coupled White-Light Interferometers", Proceedings OFS'84, pp. 361-364 (1984)

2. Ph. Dabkiewicz and R. Ulrich, "Spectral Encoding for Fiber-Optic Industrial Sensors", EFOC/LAN 85, pp. $212-$ 217 (1985)

3. C. Mariller and M. Lequime, "Fiber-Optic White-Light Birefringent Temperature Sensor", SPIE Vol. 798, Fiber Optic Sensors II, pp. 121-130 (1987)

4. C. Lecot, M. Lequime, H. Giovannini and S.J. Huard, "A New Spectral Detection Method for Fiber-Coupled Interferometers", SPIE Vol. 1169, Fiber Optic and Laser Sensors VII, pp. $73-79$ (1989)

5. M. Lequime, C. Lecot, H. Giovannini and S.J. Huard, "A Dual-Wavelength Passive Homodyne Detection Unit for Fiber-coupled White-light Interferometers", Fiber Optic Sensors IV, The Hague (1990)

6. Bertin French Patent $N^{\circ} 9000318$

7. G.W. Day and A.H. Rose, "Faraday Effect Sensors: The State of the Art", SPIE Vol. 985, 138 (1988)

8. M.N. Deeter, A. H. Rose and G.W. Day, "Faraday-effect Magnetic Field Sensors Based on Substituted Iron Garnets", Fiber Optic and Laser Sensors VIII, San José (1990)

9. H. Giovannini, M. Lequime and S.J. Huard, to be published 